\title{
Topical Gadobutrol Application Causes Fluorescence Intensity Change in RFP-expressing Tumor-Bearing Mice
}

\author{
Natalia I. Kazachkina ${ }^{1}$, Victoria V. Zherdeva ${ }^{1}{ }^{*}$, Asiya N. Saydasheva ${ }^{1}$, Irina G. Meerovich ${ }^{1}$, \\ Valery V. Tuchin ${ }^{2,3,4}$, Alexander P. Savitsky', and Alexei A. Bogdanov Jr. ${ }^{1,5}$ \\ ${ }^{1}$ Bach Institute of Biochemistry, Research Center of Biotechnology of the Russian Academy of Sciences. 33 Leninsky \\ Avenue, bld. 2, Moscow 119071, Russian Federation \\ ${ }^{2}$ Research-Educational Institute of Optics and Biophotonics, Saratov State University, 83 Astrakhanskaya str., \\ Saratov 410012, Russian Federation \\ ${ }^{3}$ Interdisciplinary Laboratory of Biophotonics, National Research Tomsk State University, 36 Lenin's Avenue, \\ Tomsk 634050, Russian Federation \\ ${ }^{4}$ Laboratory of Laser Diagnostics of Technical and Living Systems, Institute of Precision Mechanics and Control of the \\ Russian Academy of Sciences, 24 Rabochaya str., Saratov 410028, Russian Federation \\ ${ }^{5}$ Department of Radiology, University of Massachusetts Medical School, Worcester, MA 01655, USA \\ *e-mail: vjerdeva@inbi.ras.ru
}

\begin{abstract}
Optical clearing (OC) allows one to observe tissue structures and metabolic processes occurring in opaque tissues at the depths significantly exceeding the depths that can be reached without OC. Recently, we have shown that gadobutrol is a promising agent for OC of tissues in vivo. The aim of this study was to investigate the effect of time-dependence optical clearing caused by gadobutrol on intensity of fluorescent protein constitutively expressed in subcutaneous tumors in vivo. The measurements were performed in nu/nu mice bearing HEp-2 tumors expressing the red fluorescent protein TagRFP. Gadobutrol was used directly at concentrations $1.0 \mathrm{M}$ aqueous solution or as a $0.7 \mathrm{M}$ aqueous solution containing $5 \%$ dimethyl sulfoxide (DMSO). Gadobutrol was applied topically onto the skin above the tumors for $15 \mathrm{~min}$. Tissue fluorescence was measured by using in vivo planar imaging technique. It was shown that the fluorescence intensity of tumors increased by 1.1-1.5 times in different animals under the influence of gadobutrol. The increase in intensity was more pronounced in the case of $0.7 \mathrm{M}$ gadobutrol supplemented with DMSO. Apparently, the observed difference of penetration depths was due to the presence of DMSO in 0.7 M gadobutrol mixture. (C) 2021 Journal of Biomedical Photonics \& Engineering.
\end{abstract}

Keywords: fluorescence in vivo imaging; optical clearing; MRI-contrast agents; DMSO; genetically encoded fluorescent proteins; subcutaneous tumor; nude mice.

Paper \#3398 received 28 Dec 2020; revised manuscript received 28 Jan 2021; accepted for publication 29 Jan 2021; published online 27 Mar 2021. doi: 10.18287/JBPE21.07.020301.

\section{Introduction}

The planar fluorescence imaging method is based on obtaining of fluorescent images using excitation light source at epi-configuration. These methods provide a means for detecting of fluorescent signal in a living animal that reflects specific, mostly disease-related processes, such as the host immune response, inflammation, tumor growth or the presence of pathogens [1-3]. This method was successfully applied for in vivo studying of photosensitizers [4-6] or nanoparticles distribution in living animal [7, 8], for studying of various pathological processes using specific targets labeled with different fluorescent probes $[2,9]$. In the case of epifluorescence imaging fluorophore molecules 
located on the surface or very close to it provide major contribution to the total intensity of fluorescence (FI). The sensitivity limit of planar imaging methods depends on the intensity of autofluorescence of the tissue $[1,2]$.

Here, the planar fluorescence imaging method was used to study the effectiveness of optical clearing (OC). $\mathrm{OC}$ is based on reducing the structural heterogeneity of biological tissue and, as a consequence, strong light scattering, mainly due to the matching of refractive indices between condensed microstructures (cell membranes and organelles, collagen and elastin fibers of the extracellular matrix) and the interstitial fluid of tissues by applying immersion solutions, the so-called optical clearing agents (OCA) [10-14]. The OCA developed for in vivo applications should have low toxicity. Thus, it is imperative that components of OCA should be well characterized and their toxicity independently investigated and verified. Among the potential components of OCA, the most promising are the compounds which are already in the clinics and are formulated for human use. In addition, to obtain tissue landmarks that aid in FI signal interpretation it is beneficial to use multimodality approaches that provide anatomical information, e.g. a combination of fluorescence and magnetic resonance imaging (MRI). In the latter case the use of OCA with paramagnetic properties could be beneficial. One of the potential OCA is gadobutrol with optical clearing properties previously proven in experiments involving optical coherent tomography, spectral and fluorescent microscopy [15].

The aim of this work was to further investigate the OC properties of MRI contrast agent gadobutrol, including its combination with a skin permeability enhancer, and to study changes of FI of tumors expressing red fluorescent protein before and after topical application of gadobutrol to perform the optimization of OC time to achieve stable IF.

\section{Materials and methods}

\subsection{Animals and tumor model}

All animal studies were performed in accordance with national requirements for the humane treatment of experimental animals. $\mathrm{Nu} / \mathrm{Nu}$ mice (females, weight 19-21 g) were used in this work (Laboratory animal farm (Shemyakin-Ovchinnikov Institute of Bioorganic Chemistry of the RAS, Pushchino, Russia). Mice were housed in (HEPA)-filter-top cages in sterile barrier cabinet at $26{ }^{\circ} \mathrm{C}$ and $50 \%$ humidity; mice received autoclaved certified complete diet and sterile filtered drinking water ad libitum. Subcutaneous tumors in mice were obtained by impalnting HEp2-TagRFP human carcinoma cell line constitutively expressing TagRFP marker protein as described earlier [15].

\subsection{OC of tissues}

Gadobutrol (Gadovist ${ }^{\mathrm{tm}}$, Bayer, Germany) was used as OCA along (at $1.0 \mathrm{M}$ concentration as original formulation), as well as at a concentration of $0.7 \mathrm{M}$ with an addition of 5\% DMSO for improving of tissue penetration. Mice were anesthetized with a mixture of tiletamine and zolazepam ("Zoletil", Virbac Sante Animale, France) at a concentration of $25 \mathrm{mg} / \mathrm{ml}$ each and xylazine at a concentration of $20 \mathrm{mg} / \mathrm{ml}$ ("Rometar", Bioveta, a.s., Czech Republic). The mice were anesthetized with an intramuscular injection of $10 \mu \mathrm{l}$ of a mixture of zoletil-rometar $(1: 1)$.

Gadobutrol was applied for $15 \mathrm{~min}$ onto the skin above the region of subcutaneous tumor growth and covered with a hygroscopic gauze. Then the gauze was removed and OCA residue was removed with a cotton swab. The OC effect of gadobutrol were estimated by changes in initial fluorescence intensity during 60 minutes after gadobutrol application using in vivo fluorescence imaging.

\subsection{Equipment and processing of fluorescent images}

Fluorescence imaging was obtained by using an $\mathrm{iBox}^{\mathrm{TM}}$ system (UVP, USA) equipped with a motorized bodypositioning elevator and a set of filters for fluorescence excitation and image registration. The unit was equipped an automated BioLite ${ }^{\mathrm{TM}}$ light source system, which included a halogen lamp $(150 \mathrm{~W})$ and filters for excitation and registration of fluorescence and a cooled CCD camera. The system allowed to make images in white light mode (without optical filters) and fluorescence mode. In this study fluorescence was excited in the wavelength range of 502-547 nm and the registration was carried out in the range of 570-640 nm. The exposure varied from 2 to $15 \mathrm{sec}$.

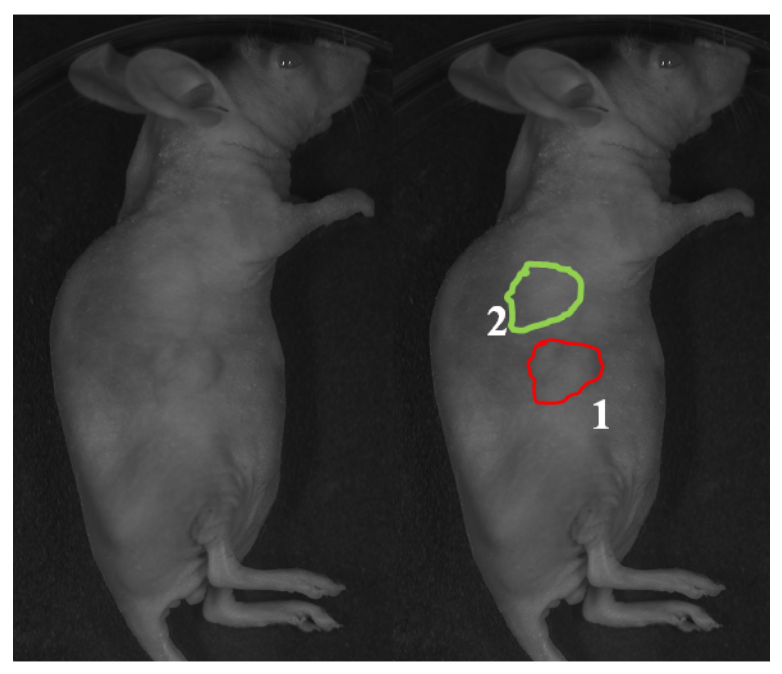

(a)

(b)

Fig. 1 Images of experimental mouse in "white light" mode (a), with the selected areas (b) of the tumor (1) and skin (2).

Fluorescence image analysis was performed using ImageJ software (NIH, USA). For image processing tumor and skin areas were highlighted (Fig. 1). The skin (background) area was set apart from the area exposed 
gadobutrol. The average fluorescence intensities (FI) of tumor and skin were calculated for each mouse with subsequent normalizing of the average FI of tumor to that of skin.

\section{Results and discussion}

Fluorescence images of mice HEp2-TagRFP tumor are shown in Fig. 2. The unevenness of red fluorescence intensity distribution throughout the tumor can be easily distinguished on these images. There was also a visible increase of FI after the application of gadobutrol, both at 1.0 M (Fig. 1a, b) as well as $0.7 \mathrm{M}$ (Fig. 1c, d) OCA. The combined results of FI measurements in all mice are shown in Tables 1 and 2.
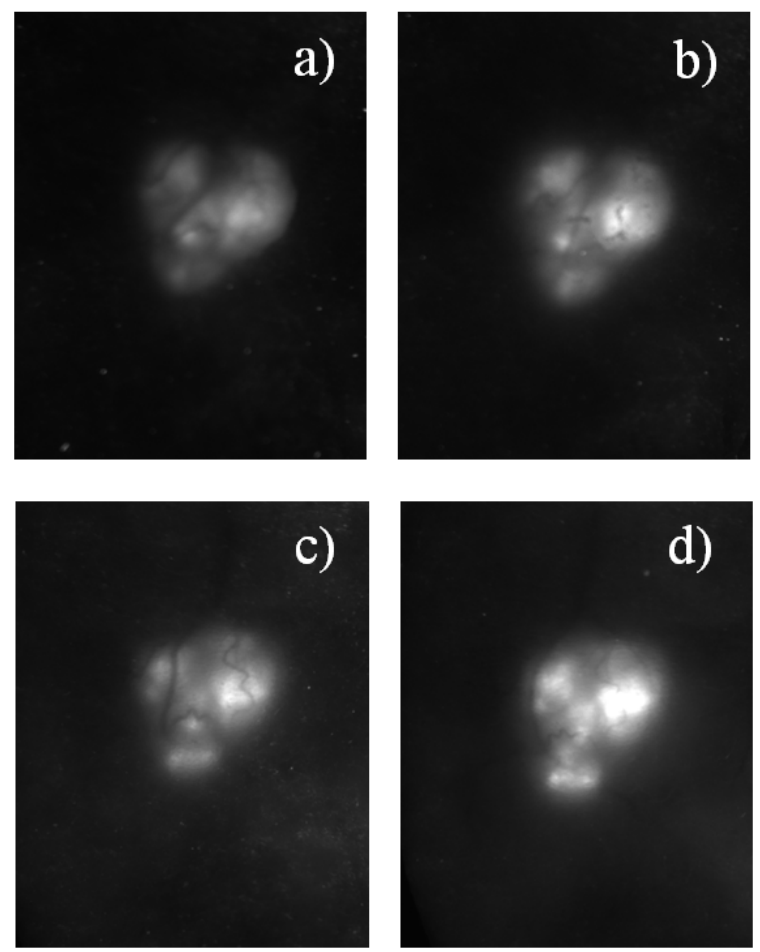

Fig. 2 Fluorescence images of HEp2-TagRFP tumor before $(\mathrm{a}, \mathrm{c})$ and $30 \mathrm{~min}$ after gadobutrol application $(\mathrm{b}, \mathrm{d})$. Exposure to $1.0 \mathrm{M}$ gadobutrol $(\mathrm{a}, \mathrm{b})$ and $0.7 \mathrm{M}$ gadobutrol with 5\% DMSO added (c, d).

As the tabulated data (Tables 1,2) show the mean values of FI of the tumor before gadobutrol application showed high levels of variability within the animal group. At the same time, various exposure times had to be used to avoid exceeding the dynamic range of the CCD detector. Under such experimental conditions the comparisons of the non-corrected mean FI values of the tumors and skin areas could not be performed. Thus, in the present study relative values were used to analyze the fluorescence signal intensity changes. Fig. 3 shows the change in FI relative to the initial value of FI (i.e. before OC).
It was shown that the fluorescence intensity of subcutaneous tumors was increased after treating the skin with gadobutrol. However, the dynamics of FI changes was dissimilar in the case of $1.0 \mathrm{M}$ gadobutrol and the OCA containing gadobutrol and DMSO.

Thus, on average, the fluorescence signal in the group of mice treated with $1.0 \mathrm{M}$ gadobutrol increased immediately after the application and then remained nearly unchanged for $1 \mathrm{~h}$. The mice treated with $0.7 \mathrm{M}$ gadobutrol supplemented with 5\% DMSO showed a steady increase of FI signal throughout the entire observation period. From the results of data analysis, it was also apparent that the fluorescence intensity of the skin was nearly unchanged. This allowed to use the fluorescence of the non-treated skin area that was not exposed to gadobutrol for FI normalization (Fig. 3).

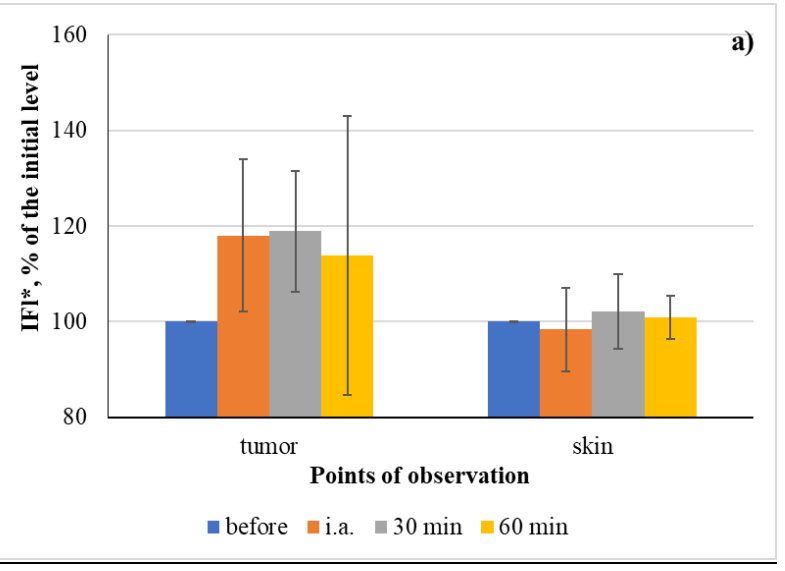

b)

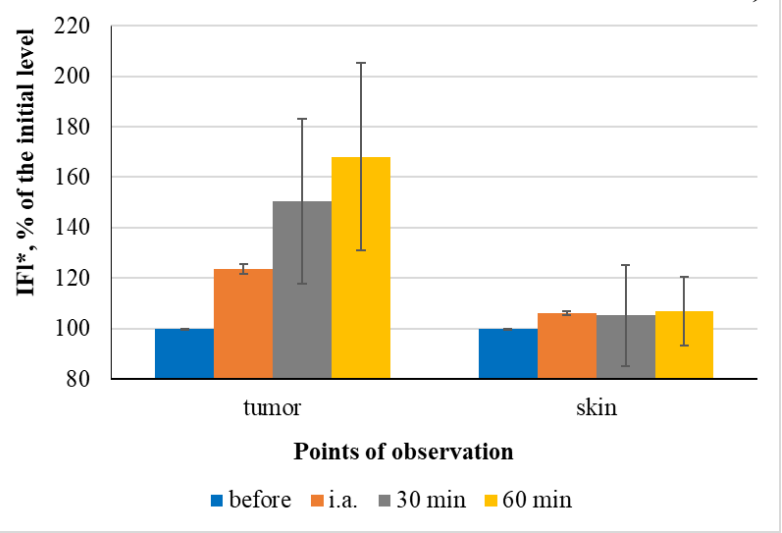

Fig. 3 Fluorescence intensity changes in tumors and skin before and after gadobutrol application. FI after application normalized on initial FI before treating the skin with gadobutrol. Gadobutrol was applied onto the area above the tumor, and the skin area was not exposed to gadobutrol: a) 1.0 M gadobutrol treatment $(\mathrm{n}=4)$; and b) $0.7 \mathrm{M}$ gadobutrol treatment with the addition of $5 \%$ DMSO $(\mathrm{n}=4$, exclude mouse \#5 data, see Table 2$)$. 
Table 1 Fluorescence intensity of HEp2-TagRFP tumors and the skin of the tumor-bearing mice treated with $1.0 \mathrm{M}$ gadobutrol.

\begin{tabular}{|c|c|c|c|c|c|c|}
\hline \multirow{3}{*}{ Mouse \# } & \multirow{3}{*}{ Exposure time, $\mathrm{s}$} & \multirow{3}{*}{ Tissue } & \multicolumn{4}{|c|}{ FI, a. u. } \\
\hline & & & \multirow{2}{*}{$\begin{array}{c}\text { Before } \\
\text { application }\end{array}$} & \multicolumn{3}{|c|}{ After application } \\
\hline & & & & $\begin{array}{c}\text { Immediately } \\
\text { after }\end{array}$ & $30 \mathrm{~min}$ & $60 \mathrm{~min}$ \\
\hline \multirow{2}{*}{1} & \multirow{2}{*}{2} & Tumor & 11995 & 16696 & 14875 & 16296 \\
\hline & & Skin & 2972 & 3213 & 3314 & 2934 \\
\hline \multirow{2}{*}{2} & \multirow{2}{*}{2} & Tumor & 12212 & 13709 & 12643 & \multirow{2}{*}{$\mathrm{n} / \mathrm{d}$} \\
\hline & & Skin & 2972 & 2647 & 2750 & \\
\hline \multirow{2}{*}{3} & \multirow{2}{*}{5} & Tumor & 14728 & 14896 & 16961 & 11895 \\
\hline & & Skin & 3218 & 3191 & 3419 & 3495 \\
\hline \multirow{2}{*}{4} & \multirow{2}{*}{2} & Tumor & 13625 & 16302 & 18117 & 17025 \\
\hline & & Skin & 2038 & 2060 & 2055 & 2096 \\
\hline
\end{tabular}

$\mathrm{n} / \mathrm{d}-$ not determined.

Table 2 Fluorescence intensity of HEp2-TagRFP tumors and the skin of the tumor-bearing mice treated with $0.7 \mathrm{M}$ gadobutrol supplemented with 5\% DMSO.

FI, a. u.

\begin{tabular}{|c|c|c|c|c|c|c|c|}
\hline \multirow{3}{*}{$\begin{array}{c}\text { Mouse } \\
\#\end{array}$} & \multirow{3}{*}{ Exposure time, $\mathrm{s}$} & \multirow{3}{*}{ Tissue } & & & & & \\
\hline & & & \multirow{2}{*}{$\begin{array}{c}\text { Before } \\
\text { application }\end{array}$} & \multicolumn{4}{|c|}{ After application } \\
\hline & & & & $\begin{array}{c}\text { Immediately } \\
\text { after }\end{array}$ & $15 \mathrm{~min}$ & $30 \mathrm{~min}$ & $60 \mathrm{~min}$ \\
\hline \multirow{2}{*}{1} & \multirow{2}{*}{2} & Tumor & 12505 & \multirow{2}{*}{$\mathrm{n} / \mathrm{d}$} & 17190 & 23948 & 24316 \\
\hline & & Skin & 2132 & & 2208 & 2781 & 2763 \\
\hline \multirow{2}{*}{2} & \multirow{2}{*}{2} & Tumor & 8790 & \multirow{2}{*}{$\mathrm{n} / \mathrm{d}$} & 12700 & 11142 & \multirow{2}{*}{$\mathrm{n} / \mathrm{d}$} \\
\hline & & Skin & 2704 & & 2705 & 2556 & \\
\hline \multirow{2}{*}{3} & \multirow{2}{*}{3} & Tumor & 23686 & 29632 & \multirow{2}{*}{$\mathrm{n} / \mathrm{d}$} & 28809 & 33588 \\
\hline & & Skin & 2735 & 2888 & & 2324 & 2310 \\
\hline \multirow{2}{*}{4} & \multirow{2}{*}{5} & Tumor & 10883 & 13319 & \multirow{2}{*}{$\mathrm{n} / \mathrm{d}$} & 17576 & \multirow{2}{*}{$\mathrm{n} / \mathrm{d}$} \\
\hline & & Skin & 2094 & 2237 & & 2324 & \\
\hline \multirow{2}{*}{5} & \multirow{2}{*}{$10-15^{*}$} & Tumor & 24611 & 22029 & \multirow{2}{*}{$\mathrm{n} / \mathrm{d}$} & 25327 & 22911 \\
\hline & & Skin & 5711 & 4163 & & 4441 & 4594 \\
\hline
\end{tabular}

$\mathrm{n} / \mathrm{d}$ - not determined; * - before application the exposure time was $15 \mathrm{~s}$, and $10 \mathrm{~s}$ afterwards.

Normalization of tumor FI to skin FI allowed performing mean value comparisons within a set of animals with limited number of observations. The obtained normalized fluorescence (Fn) values are shown in Table 3.

The presented data show that gadobutrol application led to the increase in Fn of the tumors (Table 3, Fig. 4).
Moreover, tumors treated with $0.7 \mathrm{M}$ gadobutrol with the addition of $5 \%$ DMSO showed, on average, a distinct tendency to increased Fn in the group compared to the group of animals exposed to $1.0 \mathrm{M}$ gadobutrol, 30 and 60 minutes after the optical clearing agent impact (Fig. 4). 
Table 3 The effect of gadobutrol topical application on normalized fluorescence (Fn) values of HEp2-TagRFP tumor fluorescence in various animals.

Mouse \#

Fn (FI tumor/FI skin ratio)

\begin{tabular}{|c|c|c|c|c|}
\hline & \multirow{2}{*}{$\begin{array}{l}\text { Before application } \\
\qquad \mathrm{Fn}_{0}\end{array}$} & \multicolumn{3}{|c|}{ After application, Fn } \\
\hline & & Immediately after & $30 \mathrm{~min}$ & $60 \mathrm{~min}$ \\
\hline \multicolumn{5}{|c|}{$1.0 \mathrm{M}$ gadobutrol } \\
\hline 1 & 4.04 & 5.20 & 4.49 & 5.55 \\
\hline 2 & 4.11 & 5.28 & 4.60 & $\mathrm{n} / \mathrm{d}$ \\
\hline 3 & 4.56 & 4.67 & 5.0 & 3.40 \\
\hline 4 & 6.69 & 7.92 & 8.82 & 8.12 \\
\hline Mean* & $4.85 \pm 1.25$ & $5.77 \pm 1.46$ & $5.72 \pm 2.08$ & $5.69 \pm 2.36$ \\
\hline \multicolumn{5}{|c|}{$0.7 \mathrm{M}$ gadobutrol with 5\% DMSO added } \\
\hline 1 & 3.25 & $\mathrm{n} / \mathrm{d}$ & 4.36 & $\mathrm{n} / \mathrm{d}$ \\
\hline 2 & 5.87 & $\mathrm{n} / \mathrm{d}$ & 8.61 & 8.80 \\
\hline 3 & 11.31 & 13.25 & 12.39 & 14.54 \\
\hline 4 & 3.98 & 4.61 & 3.75 & $\mathrm{n} / \mathrm{d}$ \\
\hline 5 & 4.31 & 5.29 & 5.70 & 4.99 \\
\hline Mean* & $5.74 \pm 3.26$ & $7.72 \pm 4.80$ & $6.96 \pm 3.57$ & $9.44 \pm 4.81$ \\
\hline
\end{tabular}

*data shown as mean $\pm \mathrm{SD} ; \mathrm{n} / \mathrm{d}$ - not determined

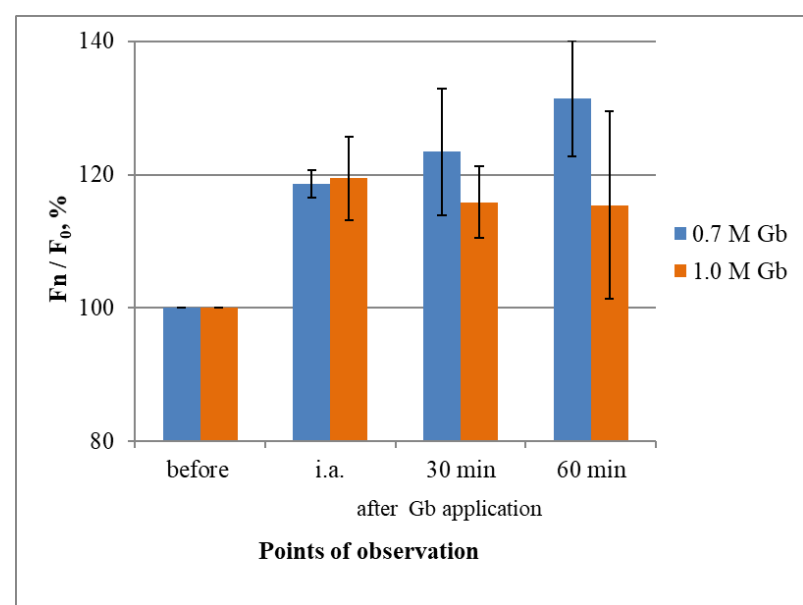

Fig. 4 The changes of HEp2 -TagRFP tumor normalized fluorescence (Fn) after topical application of two different gadobutrol $(\mathrm{Gb})$-containing solutions on the average for the group: $1.0 \mathrm{M}$ gadobutrol $(\mathrm{n}=4), 0.7 \mathrm{M}$ gadobutrol with the addition of $5 \%$ DMSO $(n=5)$; $\mathrm{F}_{0}$ - initial level of Fn (was taken as $100 \%$ ) and Fn - after gadobutrol application. Data is shown as $\mathrm{M} \pm \mathrm{SD}$. i.a. - immediately after application.

However, when considering the effect on each animal separately, it was noted that the dynamics of Fn changes varied in each animal (Fig. 5). Fn increased in most mice immediately after the application of gadobutrol, which facilitated selecting this observation period for further studies using gadobutrol as OCA. At the subsequent periods of observation, a decrease in Fn could be observed but afterwards an increase could be observed again.
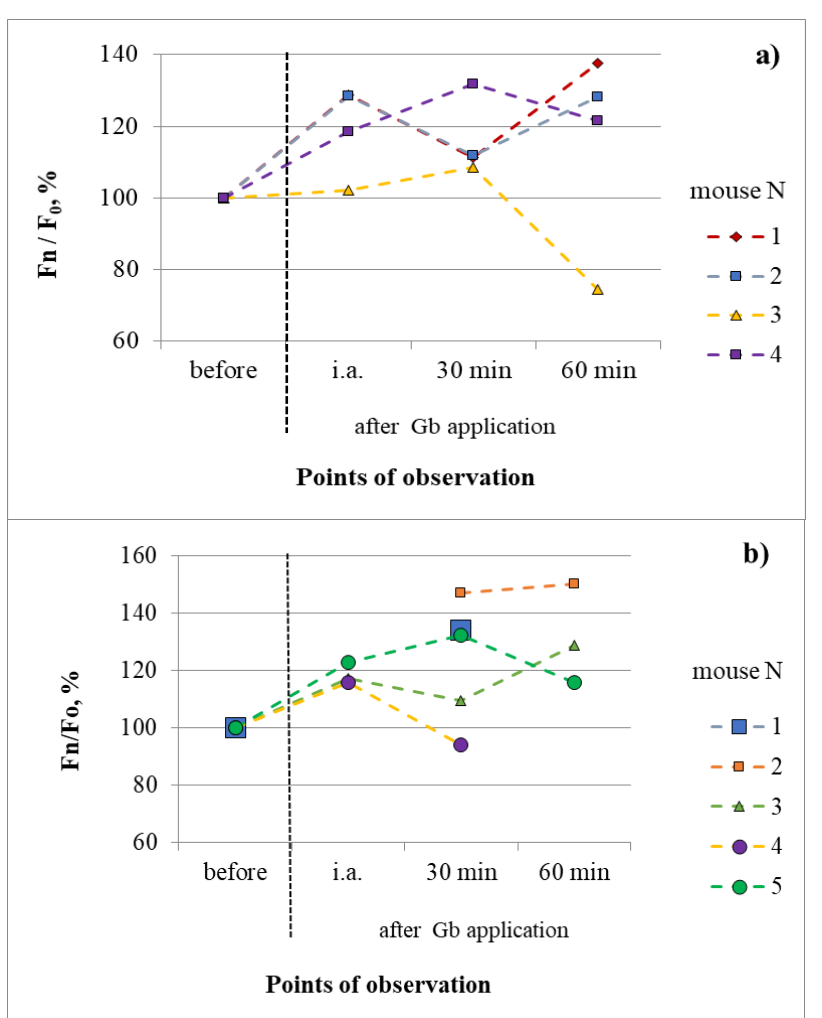

Fig. 5 The changes of the normalized fluorescence (Fn) of the HEp2-TagRFP tumor in individual animals relative to the initial level $\left(\mathrm{Fn} / \mathrm{F}_{0}\right)$ as a consequence of treating the tumors with: a) $1.0 \mathrm{M}$ gadobutrol, b) $0.7 \mathrm{M}$ gadobutrol with the addition of $5 \%$ DMSO; $\mathrm{F}_{0}$ - initial level of Fn (was taken as 100\%) and Fn-after gadobutrol application; i.a. - immediately after application. 
The oscillatory pattern of OC effect could be associated with the action of the clearing agent, which sets the water in motion both in the interstitial fluid and in the connective tissue fibers themselves. We hypothesize that the OCA penetrated into the interstitial space and caused a temporary dehydration of collagen fibers, and as a result the refractive index increased in comparison to the pre-OC levels. The resultant scattering of the tissue increased slightly but then the same effect on tissue refraction recurred as a new portion of the agent approached and equalized the refractive index again [16]. In the case of fluorescent tumor, the effects on FI could be even more complicated: a drop-in intensity and a subsequent increase may be associated with the different brightness of the luminous nodes in depth of the tissue, which become more prominent with OC. These effects could result from the heterogeneity of the tumor itself (the data will be published elsewhere, Ref. [17]). Alternatively, as we showed before [18] the FI levels should be measured in various selected areas of the tumor, and not only by integrating the whole tumor volume or individual tumor nodule.

\section{Conclusion}

Thus, the effect of OC caused by gadobutrol topical application as a potential OCA was investigated at the macroscopic level in vivo. There was a profound effect of OC on FI causing an increase of FI of HEp2 tumors expressing red fluorescent TagRFP marker protein. The OC effect of gadobutrol was associated with its transdermal penetration into the tissue after it was applied to the skin surface. This effect can be tentatively explained by the observation made in Ref. [17], where it was shown that the T1-weighted MR signal was increased after the application of the gradient echo pulse sequences in the deeper layer of the subcutaneous tissue after the application of gadobutrol/DMSO mixtures. It was also determined that the FI increase in the tumor after gadobutrol topical application persisted for a longer time period if OCA containing $0.7 \mathrm{M}$ gadobutrol was used in combination with the addition of $5 \%$ DMSO vs. $1.0 \mathrm{M}$ gadobutrol solution. We associate the mechanism of the observed OC effect of gadobutrol with a high value of its refractive index $[15,19]$, which is influenced by its high density, molecular structure and the presence of chelated polarizable gadolinium ions. The observed effects bear similarities to those of iodine-containing aromatic compounds which have high refractive indices in solutions and are used as immersion agents in many applications, including biology, mineralogy, and others [19]. This is an assumption requiring separate experimentation, which is beyond the scope of this study. At present, the results obtained allow us to draw the following conclusions:

1) both OCA under study containing gadobutrol cause OC effect when applied to the tumor growth area;

2) the most appropriate observation period is immediately after $15 \mathrm{~min}$ application for measuring fluorescence intensity after gadobutrol application;

3) $0.7 \mathrm{M}$ gadobutrol with the addition of $5 \%$ DMSO is preferable over $1.0 \mathrm{M}$ gadobutrol as it allows to observe the OC effect for a longer period of time.

\section{Disclosures}

All authors declare that there is no conflict of interests in this paper.

\section{Acknowledgements}

The work of Valery V. Tuchin was supported by the RFBR grant No. 18-52-16025.

\section{References}

1. A. Zelmer, T. H. Ward, "Noninvasive fluorescence imaging of small animals," Journal of microscopy 252(1), 8-15 (2013).

2. N. Stemmer, J. Mehnert, J. Steinbrink, and A. Wunder, "Noninvasive fluorescence imaging in animal models of stroke," Current Medicinal Chemistry 19(28), 4786-4793 (2012).

3. J. P. Miller, C. Egbulefu, J. L. Prior, M. Zhou, and S. Achilefu, "Gradient-Based Algorithm for Determining Tumor Volumes in Small Animals Using Planar Fluorescence Imaging Platform,” Tomography 2(1), 17-25 (2016).

4. I. G. Meerovich, N. I. Kazachkina, and A. P. Savitsky, "Investigation of the effect of photosensitizer Tiosense on the tumor model melKor-TurboRFP expressed red fluorescent protein,” Russian Journal of General Chemistry 85(1), 274-279 (2015).

5. E. V. Sanarova, A. V. Lantsova, A. P. Polozkova, O. L. Orlova, I. G. Meerovich, L. M. Borisova, M. P. Kiseleva, Z. S. Smirnova, N. Yu. Kul'bachevskaya, O. I. Konyaeva, and N. A. Oborotova, "Effectiveness of liposomal system of delivery of hydrophobic antineoplastic Thiosens photosensitizer," Nanotechnologies in Russia 10(5-6), 492-500 (2015).

6. A. I. Gavrina, M. V. Shirmanova, N. A. Aksenova, D. V. Yuzhakova, L. B. Snopova, A. B. Solovieva, P. S. Timashev, V. V. Dudenkova, and E. V. Zagaynova, "Photodynamic therapy of mouse tumor model using chlorin e6- polyvinyl alcohol complex," Journal of Photochemistry and Photobiology B: Biology 178, 614-622 (2018).

7. Y. Guan, T. Sun, J. Ding, and Z. Xie, "Robust organic nanoparticles for noninvasive long-term fluorescence imaging," Journal of Materials Chemistry B 7(44), 6879-6889 (2019). 
8. Y. F. Loginova, Y. F. Loginova, S. V. Dezhurov, V. V. Zherdeva, N. I. Kazachkina, M. S. Wakstein, and A. P. Savitsky, "Biodistribution and stability of CdSe core quantum dots in mouse digestive tract following per os administration: advantages of double polymer/silica coated nanocrystals," Biochemical and Biophysical Research Communications 419(1), 54-59 (2012).

9. F. Stuker, J. Ripoll, and M. Rudin, "Fluorescence Molecular Tomography: Principles and Potential for Pharmaceutical Research," Pharmaceutics 3(2), 229-274 (2011).

10. D. Zhu, K. V. Larin, Q. Luo, and V. V. Tuchin, "Recent progress in tissue optical clearing," Laser \& Photonics Reviews 7(5), 732-757 (2013).

11. D. S. Richardson, J. W. Lichtman, “Clarifying tissue clearing," Cell 162(2), 246-257 (2015).

12. P. Matryba, L. Kaczmarek, and J. Gołab, “Advances in ex situ tissue optical clearing," Laser \& Photonics Reviews 13(8), 1800292 (2019).

13. I. Costantini, R. Cicchi, L. Silvestri, F. Vanzi, and F. S. Pavone, "In-vivo and ex-vivo optical clearing methods for biological tissues: Review," Biomedical Optics Express 10(10), 5251-5267 (2019).

14. A. Yu. Sdobnov, J. Lademann, M. E. Darvin, and V. V. Tuchin, "Methods for Optical Skin Clearing in Molecular Optical Imaging in Dermatology," Biochemistry 84(Suppl 1), S144-S158 (2019).

15. D. K. Tuchina, I. G. Meerovich, O. A. Sindeeva, V. V. Zherdeva, A. P. Savitsky, A. A. Bogdanov Jr., and V. V. Tuchin, "Magnetic resonance contrast agents in optical clearing: Prospects for multimodal tissue imaging," Journal of Biophotonics 13(11), e201960249 (2020).

16. Q. Xie, N. Zeng, Y. Huang, V. V. Tuchin, and H. Ma, "Study on the tissue clearing process using different agents by Mueller matrix microscope,” Biomedical Optics Express 10(7), 3269 (2019).

17. A. A. Bogdanov Jr., N. I. Kazachkina, V. V. Zherdeva, I. G. Meerovich, D. K. Tuchina, I. D. Solovyev, A. P. Savitsky, and V. V. Tuchin, "Magnetic resonance imaging study of diamagnetic and paramagnetic agents for optical clearing of tumor-specific fluorescent signal in vivo," Chapter 25 in Tissue optical clearing: new prospects in optical imaging, D. Zhu, E. Genina, and V. Tuchin (Eds.), CRC Press, Boca Raton, FL (2021) [In press].

18. D. K. Tuchina, I. G. Meerovich, O. A. Sindeeva, V. V. Zherdeva, N. I. Kazachkina, I. D. Solov'ev, A. P. Savitsky, A. A. Bogdanov Jr., and V. V. Tuchin, "Prospects for multimodal imaging of biological tissues using fluorescence imaging," Quantum Electronics 51(2), 104-117 (2021).

19. K. Bai, J. Katz, "On the refractive index of sodium iodide solutions for index matching in PIV," Experiments in Fluids 55, 1704 (2014). 